Muséologies

Les cahiers d'études supérieures

muséologies

\title{
Exposition permanente, expositions temporaires : deux façons de parler sculpture au Petit Palais, Musée des Beaux-Arts de la Ville de Paris
}

\section{Amélie Simier}

Volume 3, numéro 2, printemps 2009

URI : https://id.erudit.org/iderudit/1033566ar

DOI : https://doi.org/10.7202/1033566ar

Aller au sommaire du numéro

Éditeur(s)

Association Québécoise de Promotion des Recherches Étudiantes en Muséologie (AQPREM)

ISSN

1718-5181 (imprimé) 1929-7815 (numérique)

Découvrir la revue

Citer cet article

Simier, A. (2009). Exposition permanente, expositions temporaires : deux façons de parler sculpture au Petit Palais, Musée des Beaux-Arts de la Ville de Paris. Muséologies, 3(2), 112-121. https://doi.org/10.7202/1033566ar d'utilisation que vous pouvez consulter en ligne. 
Article huit

Exposition permanente, expositions temporaires:

deux façons de parler sculpture au Petit Palais,

Musée des Beaux-Arts de la Ville de Paris

AMÉLIE SIMIER EST CONSERVATEUR DU PATRIMOINE, CHARGEEE DES SCULPTURES ET MÉDAILLES AU PETIT PALAIS, MUSÉE DES BEAUX-ARTS DE LA VILLE DE PARIS. ELLE PUBLIE RÉGULIEREMENT DES ARTICLES SUR LA SCULPTURE DU DERNIER TIERS DU XIX' SIECLE. EN 2007-2008, ELLE A ASSURÉ AU PETIT PALAIS LE COMMISSARIAT DE LEXPOSITION LA MATIERE DE L'ETRANGE JEAN CARRIÈS 1855-1894. À CETTE OCCASION, ELLE A PUBLÉ LE CATALOGUE SOMMAIRE DES CEUVRES DE CARRIES AU PETIT PALAIS, EN COLLABORATION AVEC DOMINIQUE MOREL. ELLE PRÉPARE PRÉSENTEMENT LE CATALOGUE RAISONNÉ DES CEUVRES DU SCULPTEUR JULES DALOU CONSERVÉES AU PETIT PALAIS. ELLE A ÉTÉ CO-COMMISSAIRE DEXPOSITIONS ORGANISÉES PAR LE PETIT PALAIS À LÉTRANGER, DONT PARIS 1900 DANS LES COLLECTIONS DU PETIT PALAIS AU MUSÉE DES BEAUX-ARTS DE QUÉBEC (2007-2008). ELLE ENSEIGNE PAR AILLEURS L'HISTOIRE DE LART DU XIX SIĖCLEÀ L'ÉCOLE DU LOUVRE DEPUIS QUATRE ANS. [ametie.smier@paris.fr] 
Le Petit Palais, Musée des Beaux-Arts de la Ville de Paris, est un édifice séduisant situé sur le Coursla-Reine (dans le VIII arrondissement) dans le prolongement des Champs-Elysées. Il a été construit à l'occasion de l'Exposition universelle de 1900, puis affecté aux collections de beaux-arts de la ville de Paris. À son ouverture en décembre 1902, il présentait les sculptures acquises par la Ville dans deux galeries encadrant l'entrée principale, posées sur des socles hauts, éclairées d'un jour latéral. L'aménagement des salles d'expositions a évolué au fil de l'arrivée d'œuvres nouvelles dans les collections. Ainsi, pour la sculpture, une salle dédiée au sculpteur et céramiste Jean Carriès (1848-1894) a été créée dès 1905, à l'arrivée du don de son fonds d'atelier par Georges Hoentschel. Les œuvres y étaient exposées dans leur ensemble, sur sellettes, sous vitrines ou suspendues aux murs, sans autre accompagnement pédagogique que la publication régulière d'un catalogue sommaire des collections du musée.

Dès l'ouverture, le musée a aussi été envisagé comme un lieu destiné aux manifestations et aux expositions temporaires. On se souvient que le premier Salon d'automne s'y est tenu en 1903. Ces dernières années, une active politique d'expositions a fidélisé un public d'amateurs - le même public que celui du Grand Palais, avec lequel on confond souvent le Petit Palais.

Le musée a bénéficié tout récemment d'une rénovation importante, menée par le cabinet Chaix \& Morel et Associés. La rénovation s'est appuyée sur un projet scientifique et culturel établi par le directeur du musée, Gilles Chazal. Le constat était le suivant: le musée contient des collections éclectiques, qui couvrent une large période (de l'Antiquité au début du XXe siècle) et des techniques différentes (dessin, gravure, peinture, sculpture, objets d'art...). 


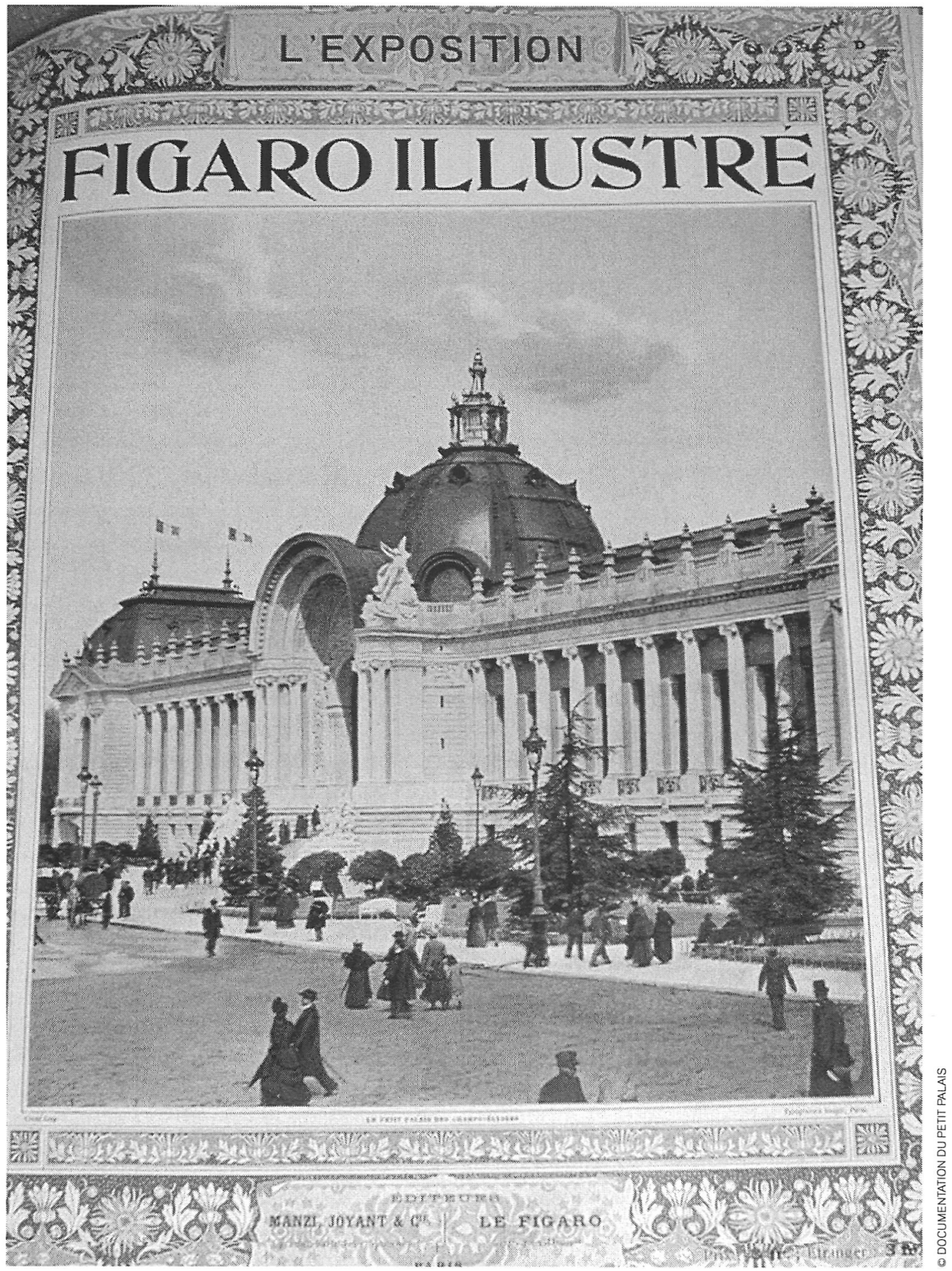

Le Petit Palais à l'Exposition universelle de 1900, vue extérieure. Première de couverture du Figaro Illustré, 1900. 
Il est admirablement placé dans Paris, mais il est en concurrence directe avec l'offre culturelle pléthorique de la capitale. Il ne peut rivaliser avec le projet encyclopédique de ses grands voisins : le musée du Louvre ou celui d'Orsay. Mais il peut mettre en valeur ses points forts : ainsi le charme de son architecture 1900 et les ensembles constitués par des collectionneurs amoureux du musée.

Le choix de présentation des objets favorise désormais la délectation, sans ignorer l'éducation. On y trouve une présentation esthétique des œuvres majeures, plutôt qu'une présentation exhaustive des collections, toutes techniques mises en résonnance. Philippe Chaix, l'architecte qui a suivi tout particulièrement le chantier, a privilégié le travail sur les volumes, l'espace et la clarté.

La scénographie a aussi vocation pédagogique sur le principe des " confrontations suggestives ", une œuvre ou un ensemble d'œuvres en éclairant d'autres. Ainsi la salle Renaissance est voisine de la salle des Antiques. Par ailleurs, des outils pédagogiques ont été développés par la conservation: fiches de salles, cartels et, plus récemment, audioguides à destination des adultes et des enfants.

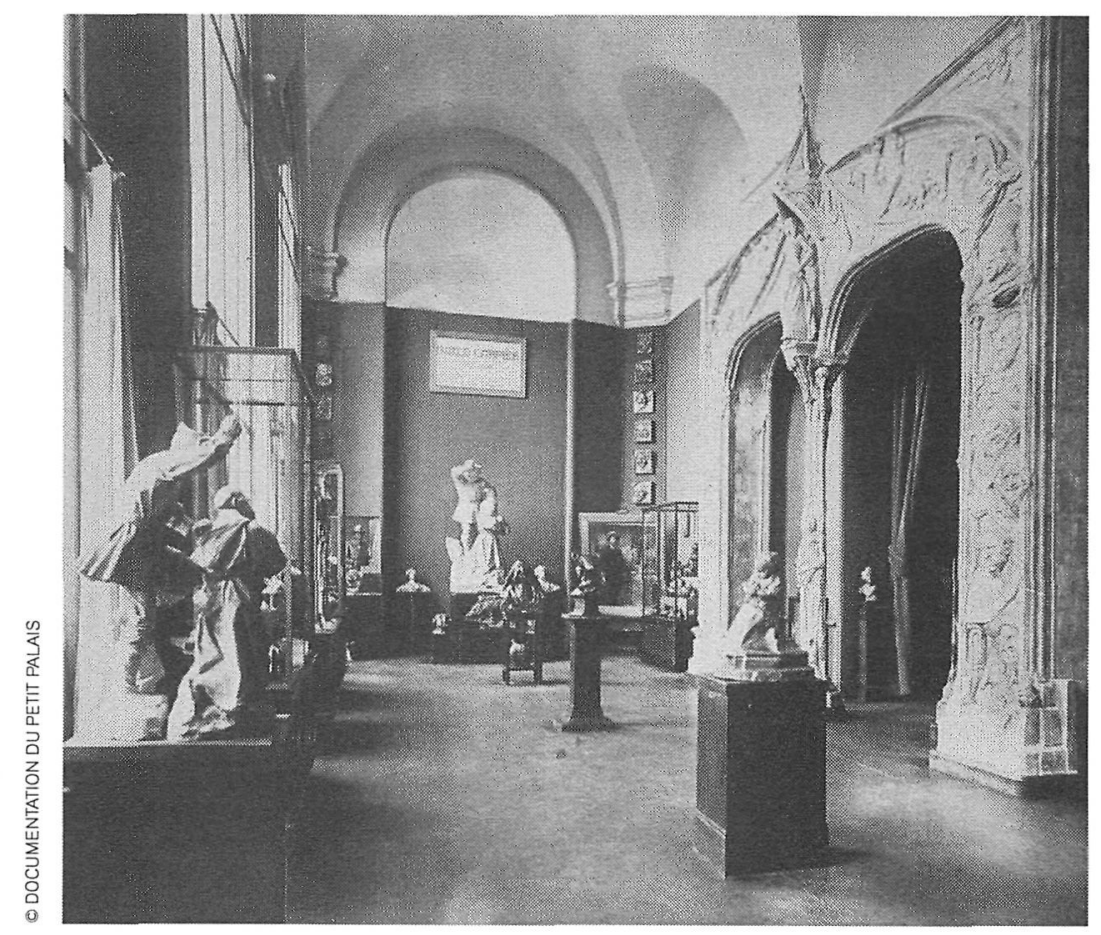


Le Service éducatif et culturel assure un accompagnement pédagogique important, par des visites guidées, contées ou animées, et des ateliers encadrés par des plasticiens. Il est ouvert aux individuels, aux familles, comme aux classes qui viennent en nombre.

Dans ce cadre général, penchons-nous sur le cas de la sculpture dans les collections permanentes du Petit Palais. Le fonds de sculpture, dont j'ai la charge comme conservateur, est important : 2842 sculptures et médailles, pour 151 sculptures exposées actuellement. La période la mieux représentée est la seconde moitié du XIX ${ }^{e}$ siècle. Certains fonds sont sans équivalent dans d'autres collections publiques : les fonds Jean Carriès, Jules Dalou et le fonds d'esquisses et de modèles pour les monuments publics parisiens.

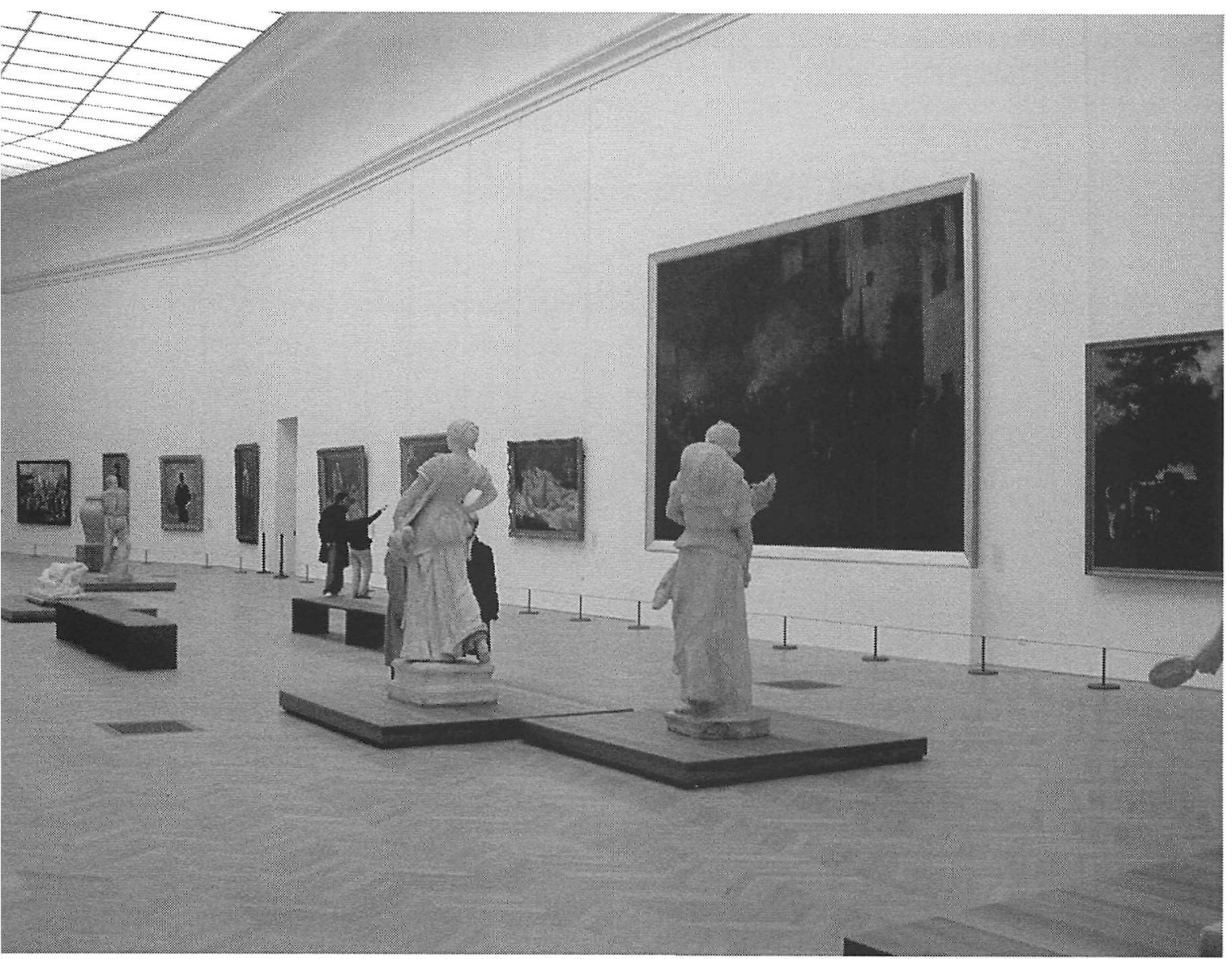


Tel que souligné précédemment, une confrontation s'opère donc entre sculpture et autres arts. Bien souvent, la sculpture, en raison de son volume, occupe le centre des salles et dialogue avec la peinture et les objets d'art. Prenons l'exemple de la Galerie des grands formats : au centre d'un vaste espace à éclairage zénithal consacré aux grands formats de la seconde moitié du XIX ${ }^{e}$ siècle, peintures et sculptures, se trouvent dix œuvres: des statues de marbre, achevées (Auguste Clésinger, AiméJules Dalou, Louis Ernest Barrias), ou des esquisses et des modèles en plâtre de monuments publics parisiens (Émile Chatrousse, Henri Chapu, Jules Félix Coutan, Aimé Jules Dalou). Elles renvoient aux tableaux mis à proximité:la Bacchante de Clésinger, scandale du Salon de 1849, est ainsi proche du Sommeil de Gustave Courbet, œuvre sulfureuse de 1866 sur un thème voisin... Pour que la délectation prime, les œuvres soigneusement espacées ont été posées sur des socles bas, sans vitre, et les cartels et les fiches pédagogiques renvoyés sur les murs. Dans un souci de sécurité, les socles ont été élargis pour servir de mise à distance. Le tout est le fruit d'un dialogue constant, parfois vif, entre la maîtrise d'œuvre (l'équipe de Philippe Chaix), la maîtrise d'ouvrage (la Direction de l'architecture et du patrimoine) et la conservation du musée. L'espace ainsi créé est séduisant, il incite à la déambulation et... force le visiteur le plus réfractaire à la sculpture à la regarder ! Celui qui veut en savoir davantage peut se référer aux visites guidées et aux instruments de visite fournis.

Par ailleurs, des salles monographiques, insérées dans le parcours, sont consacrées aux sculpteurs majeurs: Jean-Baptiste Carpeaux, Jean Carriès et Aimé Jules Dalou. Elles sont aussi l'occasion de rendre hommage aux donateurs du musée : la salle Carriès porte le nom du généreux donateur du fonds, Georges Hoentschel.

Les expositions temporaires permettent de présenter au public certaines des œuvres habituellement conservées en réserve. Pour l'automne 2007, notre équipe a conçu une exposition intitulée La matière de l'étrange - Jean Carriès 1855-1894, consacrée à un artiste, sculpteur et céramiste fascinant, inconnu du grand public, à partir du riche fonds du Petit Palais, dont une quarantaine d'œuvres sont exposées en permanence dans un espace un peu contraint (j'en étais l'un des commissaires). Des 200 œuvres exposées temporairement, $95 \%$ provenaient des collections du musée; un bon nombre était jusque-là demeuré dans les réserves. Plâtres, cires, terres, bronzes, grès émaillés, pots, bustes, reliefs, rondes-bosses... autant d'œuvres réputées moins accessibles pour le visiteur. 
Le Petit Palais ne s'est pas orienté vers une réflexion sur les publics visés par des expositions spécifiques: nous considérons que nous nous adressons à tous. Mais, instinctivement, nous savions que nous attirerions les historiens de l'art en restituant pour la première fois le grand projet inachevé de la vie de l'artiste, sa Porte monumentale, ainsi que les céramistes, pour qui les recherches expérimentales d'émaux chatoyants de Carriès sont admirables. Nous comptions attirer les enfants en cette période de vacances scolaires, par l'univers grotesque de l'artiste - masques et créatures imaginaires. Nous espérions en outre faire venir un plus large public en rendant sensible la "cuisine" technique qui présidait aux créations de Carriès.

De façon générale, nous avons conçu un parcours pédagogique. D’abord, en racontant une histoire, à l'aide de la scénographie inventive de Philippe Maffre, l'architecte retenu. Les salles, divisées en chapitres, suivaient la vie de Carriès, de la misère à la gloire et à la mort à 39 ans. L'atmosphère reposait sur les modulations de couleurs et de lumières. Le point d'orgue jouait de la reconstitution illusionniste, évoquant le chef-d'œuvre inachevé. Par ailleurs, nous avons donné aux visiteurs des outils d'appréhension pédagogiques et ludiques, conçus dans une étroite collaboration entre le scénographe, les commissaires et le service éducatif et culturel.

Ainsi la première salle, "Dans l'atelier ", dédiée aux secrets de l'atelier, comportait, entre autres, une table tactile, faite de reproductions partielles d'un carreau de Carriès, dans tous les matériaux utilisés par l'artiste. Ces moulages avaient été réalisés par Damien Granelle, plasticien intervenant au Petit Palais dans le cadre d'ateliers. Les reproductions étaient partiellement protégées, partiellement accessibles au toucher. On pouvait donc développer un discours, conçu par Fabienne Cousin, la responsable du Service éducatif, autour de ce grand problème des musées : « toucher ou ne pas toucher ". Visiblement, au bout de deux jours, la cire, le plâtre, le plâtre patiné accessibles étaient abîmés... Le véritable carreau de grès émaillé, choisi avec l'aide d'une restauratrice pour sa solidité, était soclé sur la même table et, exceptionnellement, touchable lui aussi : les visiteurs n'ont pourtant guère osé le toucher...

Les jeunes visiteurs recevaient un carnet de parcours ludique, conçu par Catherine André du Service éducatif, intitulé "Sur les pas de la grenouille» (un animal fétiche de Carriès). Les enfants étaient invités à chercher les traces de grenouille posées au sol dans le parcours, autant de lieux où le carnet les incitait à une activité. 


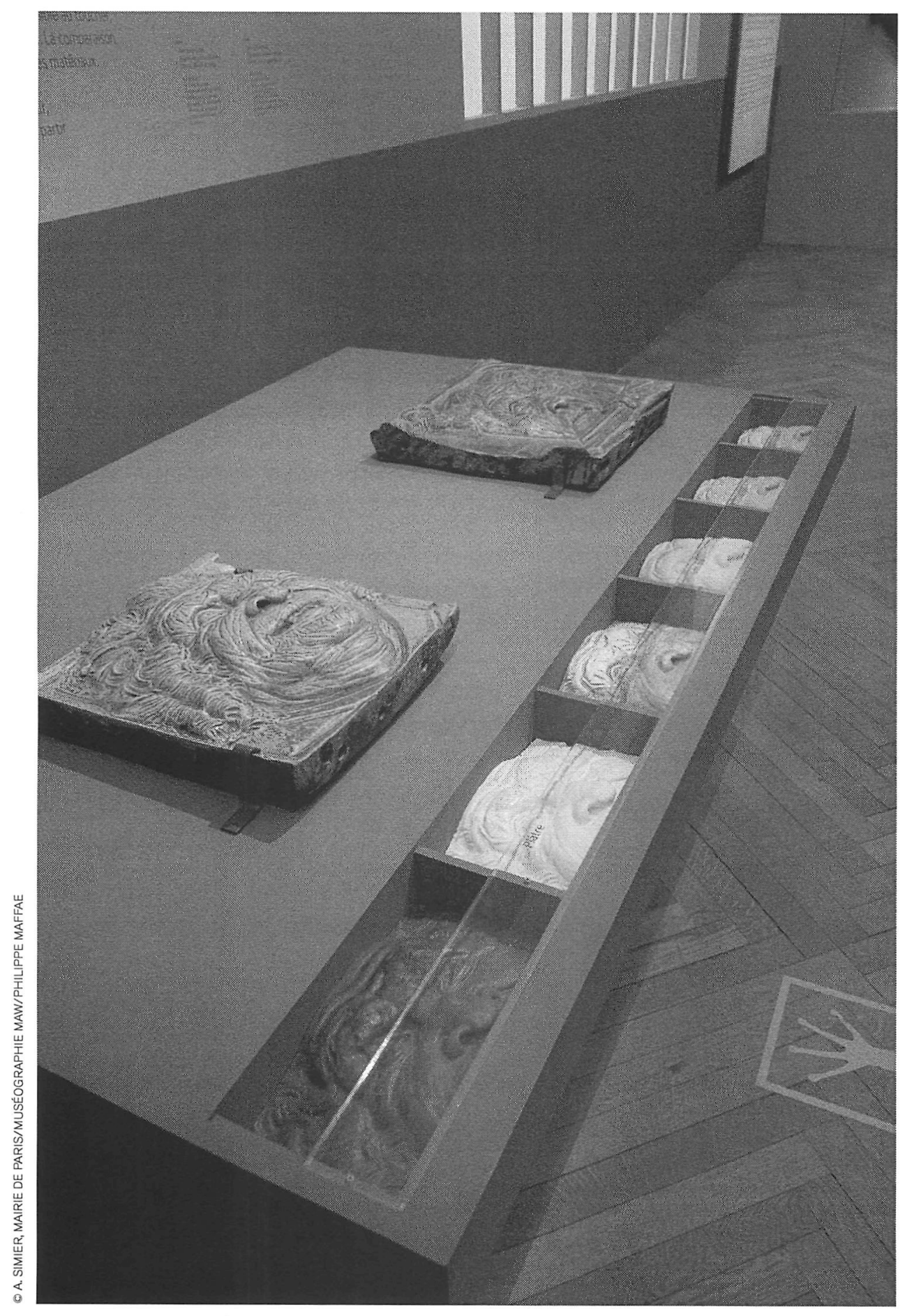

Petit Palais, exposition temporaire "La matière de l'étrange. Jean Carriès 1855-1894": "Dans l'atelier", la table tactile, moulages Damien 2007. 
Des contes, des visites, des ateliers de création autour du masque ou de l'animal fantastique étaient proposés aux visiteurs. L'exposition s'accompagnait d'un catalogue scientifique (en vente à $55 €$ ), d'un "Petit Guide» $(2,50 €)$ et d'un ouvrage très illustré de la collection Découverte Gallimard $(8,40 €)$. Chaque salle était expliquée par de longs panneaux pédagogiques.

L'exposition a connu un succès mitigé: très appréciée des spécialistes enthousiastes, largement fréquentée par les enfants, elle n'a pas su attirer le plus large public, peut-être insuffisamment informé, frileux devant des œuvres étranges et un artiste inconnu, sans doute sursollicité par la riche programmation culturelle parisienne. Mais le succès de nos efforts de pédagogie, lui, me paraît tangible. Et ce que nous avons crée ensemble autour de cette exposition est utilisé aujourd'hui encore dans les activités du Service éducatif du musée, puisque s'y trouve, en permanence, une salle, certes plus réduite, dédiée à Jean Carriès.

Les expositions temporaires me semblent être le lieu idéal de l'expérimentation. Et comme conservateur de sculpture, je suis personnellement convaincue qu'il faut accompagner l'exposition de ces objets peu regardés dans la vie de tous les jours d'un appareil pédagogique important pour qu'ils soient mieux appréciés. Dans cette optique, quoi de mieux que ces ateliers de musées qui accueillent les enfants venus avec leurs parents, avec leur classe ou leur centre de loisir, pour sensibiliser les plus petits à un art qu'ils goûteront mieux une fois grands? 


\section{Summary}

[Translated by Micheline Giroux-Aubin ]

The Petit Palais, Musée des Beaux-Arts de la Ville de Paris, constructed in 1900, has reopened in December 2005 after a major renovation operation. The new permanent collections itinerary proposes a combination of paintings, sculptures, works of art, and antiques, at the centre of chronological entities, all these arts mutually shedding light on one another for the greatest pleasure of the public. Sculptures, although quantitatively limited, hold an important place.

In the autumn of 2007, the Petit Palais hosted a temporary exhibition, created by the museum and dedicated to the sculptor and ceramist Jean Carriès. That was the occasion to design a specific scenography as well as stimulating educational experiences aimed at introducing essential keys to understand artists, materials, and techniques little known by visitors. Sculpture, an art sometimes difficult to understand in these days, particularly benefits from a specialized accompaniment during the visit. 